\title{
Rotor Initial Position Estimation Based on sDFT for Electrically Excited Synchronous Motors
}

\author{
Qing-qing Yuan*, Xiao-jie $\mathrm{Wu}^{\dagger}$, and Peng Dai ${ }^{*}$ \\ ${ }^{* \dagger}$ Dept. of Information and Electronic Engineering, China University of Mining and Technology, Xu Zhou, China
}

\begin{abstract}
Rotor initial position is an important factor affecting the control performance of electrically excited synchronous motors. This study presents a novel method for estimating rotor initial position based on sliding discrete Fourier transform (sDFT). By injecting an ac excitation into the rotor winding, an induced voltage is generated in stator windings. Through this voltage, the stator flux can be obtained using a pure integral voltage model. Considering the influence from a dc bias and an integral initial value, we adopt the sDFT to extract the fundamental flux component. A quadrant identification model is designed to realize the accurate estimation of the rotor initial position. The sDFT and high-pass filter, DFT, are compared in detail, and the contrast between dc excitation and ac injection is determined. Simulation and experimental results verify that this type of novel method can eliminate the influence of dc bias and other adverse factors, as well as provide a basis for the control of motor drives.
\end{abstract}

Key words: Electrically excited synchronous motors, Rotor initial position, Sliding discrete Fourier transform, Quadrant identification

\section{INTRODUCTION}

Electrically excited synchronous motors (EESMs) are widely applied in high-power industrial drives, such as metal rolling, mine hoisting, ship propulsion, and locomotive traction [1-4] because of their high efficiency and adjustable power factor. The stator frequency of EESM is zero $f_{\mathrm{s}}=0$ $\mathrm{Hz}$ ) at the starting time. An incorrect estimation of the rotor initial position at this time can affect the success of the start-up and influence the accuracy of the flux observing [5]-[7].

Numerous studies have been conducted to estimate rotor initial position. The traditional method involved injecting dc excitation into the rotor winding while the stator windings were not electrified, followed by the induced voltage and the flux amplitude. The corresponding angle can be obtained by a pure integral voltage model. However, the traditional method has several disadvantages, including: 1) fast attenuation of the induced voltage because of dc excitation; 2) a dc bias and an integral value resulting from a pure integral voltage

Manuscript received Jan. 7, 2014; accepted Mar. 14, 2014

Recommended for publication by Associate Editor Kwang-Woon Lee.

${ }^{\dagger}$ Corresponding Author: cumtxz1215@163.com

Tel: +86-516-83885667, Fax: +86-516-83885667, China University of Mining and Technology

*Dept. of Information and Electronic Engineering, China University of Mining and Technology, China model; and 3) other factors, such as inverter nonlinearity, dead zone, and high frequency interference [6]-[8]. The use of ac excitation as a replacement can solve the fast attenuation problem, but the integral initial value and the dc bias error persist. An improved voltage model can only address the issue of initial value.

High-frequency signal injection [9]-[11], in which filter performance is an important factor that affects position estimation, is another common approach. Compared with discrete Fourier transform (DFT), sliding DFT (sDFT) can extract a signal spectrum with faster arithmetic speed and simpler implementation [12].

This paper presents a novel estimation method for the rotor initial position of EESM. First, an ac excitation is injected into the rotor winding. Second, flux components can be obtained with the induced voltage through a pure integral voltage model. Third, the fundamental flux is extracted along with the rotor initial position as SDFT settles the dc bias and the initial integral value.

The paper is organized as follows: Section II summarizes the problems with the traditional method. Section III describes the principle of sDFT and its difference from high-pass filter (HPF) and DFT. Section IV proposes a novel method based on sDFT with ac rather than dc excitation. Simulation and experimental results are shown in Section V, and conclusions are provided in Section VI. 


\section{PRoblems With the TRAditional Method}

A sine induced voltage, which is generated in the stator windings, can avoid fast attenuation with an ac excitation injected into the rotor winding of EESM. The stator voltage vector should be perpendicular to the stator flux under ideal conditions. Once dc bias error occurs during the integration, the relationship among the stator-induced voltage, flux, and rotor initial position in the two-phase static coordinates are as follows:

$$
\begin{gathered}
e_{\mathrm{s} \alpha}= \pm a \cdot \cos \theta+\beta_{\mathrm{s} \alpha} \\
e_{\mathrm{s} \beta}= \pm b \cdot \cos \theta+\beta_{\mathrm{s} \beta} \\
\psi_{\mathrm{s} \alpha}=\int e_{\mathrm{s} \alpha} \mathrm{d} t= \pm \frac{a}{\omega} \sin \theta+\beta_{\mathrm{s} \alpha} T \\
\psi_{\mathrm{s} \beta}=\int e_{\mathrm{s} \beta} \mathrm{d} t= \pm \frac{b}{\omega} \sin \theta+\beta_{\mathrm{s} \beta} T \\
\varphi=\arctan \frac{\psi_{\mathrm{s} \beta}}{\psi_{\mathrm{s} \alpha}}
\end{gathered}
$$

where, $\beta_{\mathrm{s} a}, \beta_{\mathrm{s} \beta}$ are unknown dc biases; $a$ and $b$ represent the voltage amplitudes; $\theta$ and $\omega$ are the angle and angular frequencies of the induced cosine voltage, respectively, with $T$ denoting the integral cycle; $\psi_{\mathrm{s} \alpha}, \psi_{\mathrm{s} \beta}$ are the flux components resulting from the integral voltage model; and $\varphi$ is the flux angle, which also refers to the rotor initial position.

In an actual system, $a \gg \beta_{\mathrm{s} \alpha}, \mathrm{b} \gg \beta_{\mathrm{s} \beta}$; however, a tiny error can result in a large derivation after integration. When $\theta=\pi$, $2 \pi, 3 \pi \ldots$, the stator flux in $\alpha$ coordinate is as follows:

$$
\psi_{\mathrm{s} \alpha}=\beta_{\mathrm{s} \alpha} T_{z}
$$

where $T_{\mathrm{z}}$ represents the cycle when the voltage crosses zero.

Similarly, when $\theta=\pi, 2 \pi, 3 \pi \ldots$, the stator flux in the $\beta$ coordinate is shown as

$$
\psi_{\mathrm{s} \beta}=\beta_{\mathrm{s} \beta} T_{z}
$$

Aside from the dc bias, dead zone and inverter nonlinearity can cause an incorrect estimation of the rotor initial position.

\section{PRINCIPLE OF SDFT}

\section{A. Basic Principle of $s D F T$}

The sDFT is a recursive implementation of the DFT algorithm, which is often used to calculate the spectrum components of a finite-length signal with low computational cost [12]-[14].

Given a continuous signal in time domain $x(t)$, the fundamental frequency is $f_{0}$. With discrete sampling (sampling frequency is $f_{\mathrm{s}}$ ), this continuous signal can be transformed into a finite length sequence $x(n)$, with the length as $N=f_{\mathrm{s}} / f_{0}$. The DFT of $x(n)$ is

$$
X(k)=\operatorname{DFT}[x(n)]=\sum_{n=0}^{N-1} x(n) W_{N}^{n k},(0 \leq k \leq N-1)
$$

where $W_{N}=\mathrm{e}^{-\mathrm{j} 2 \pi / N}$

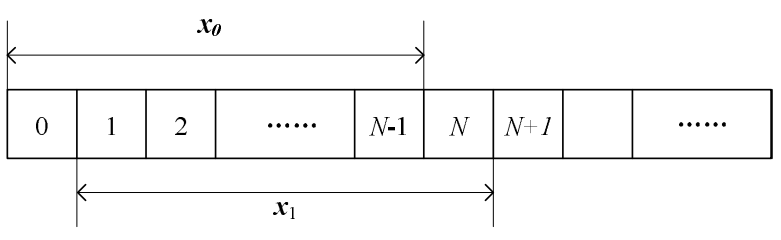

Fig. 1. Data graphics of $x_{0}(n)$ and $x_{1}(n)$.

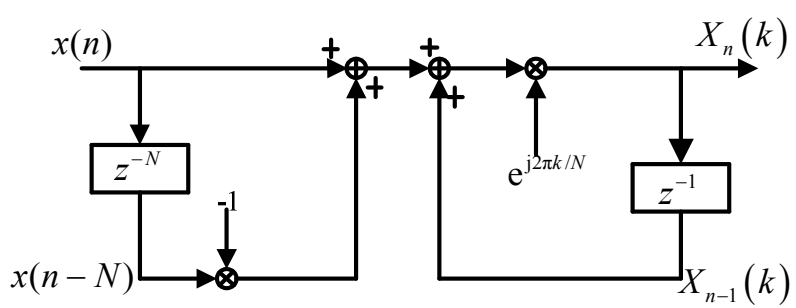

Fig. 2. Implementation structure of sDFT in the z-domain.

Equation (6) can be expanded as follows:

$X(k)=x(0)+x(1) \mathrm{e}^{-\mathrm{j} \frac{2 \pi k}{N}}+x(2) \mathrm{e}^{-\mathrm{j} \frac{2 \pi k * 2}{N}}+\cdots+x(N-1) \mathrm{e}^{-\mathrm{j} \frac{2 \pi k *(N-1)}{N}}$

Equation (7) requires $N$ data to extract the fundamental component, which adversely influences calculation speed.

Assuming that we have two finite-length sequences $x_{0}(n)$ and $x_{1}(n)$, the lengths of which are both $N$, the relationship between $x_{0}(n)$ and $x_{1}(n)$ is shown in Fig. 1 .

The DFTs of the two sequences are $X_{0}(k)$ and $X_{1}(k)$.

$$
\begin{aligned}
X_{0}(k)= & x(0)+x(1) \mathrm{e}^{-\mathrm{j} \frac{2 \pi k}{N}}+x(2) \mathrm{e}^{-\mathrm{j} \frac{2 \pi k^{*} 2}{N}}+\cdots \\
& +x(N-1) \mathrm{e}^{-\mathrm{j} \frac{2 \pi k *(N-1)}{N}} \\
X_{1}(k)= & x(1)+x(2) \mathrm{e}^{-\mathrm{j} \frac{2 \pi k}{N}}+x(3) \mathrm{e}^{-\mathrm{j} \frac{2 \pi k^{*} 2}{N}}+\cdots \\
& +x(N) \mathrm{e}^{-\mathrm{j} \frac{2 \pi k *(N-1)}{N}}
\end{aligned}
$$

Equation (9) indicates that the DFT of $x_{1}(n)$ takes new sampled data $x_{1}(N+1)$ as a replacement for $x_{0}(0)$. Substituting Equation (8) into Equation (9), $X_{1}(k)$ can be re-written as

$$
\begin{aligned}
X_{1}(k) & =\left[X_{0}(k)-x(0)\right] \mathrm{e}^{\mathrm{j} \frac{2 \pi k}{N}}+x(N) \mathrm{e}^{-\mathrm{j} \frac{2 \pi k *(N-1)}{N}} \\
& =\left[X_{0}(k)-x(0)+x(N)\right] \mathrm{e}^{\mathrm{j} \frac{2 \pi k}{N}}
\end{aligned}
$$

Equation (10) shows that $X_{1}(k)$ can calculated only by using the DFT of $x_{0}(n)$, that is, $X_{0}(k)$, and $x(0), x(N)$, along with a simple phase-shift computation.

The transfer function of sDFT that extracts $k$-harmonic in the z-domain is presented in Equation (11), and its structure is shown in Fig. 2.

$$
H_{\text {SDFT }}(z)=\frac{\left(1-z^{-N}\right) e^{\mathrm{j} 2 \pi k / N}}{1-e^{\mathrm{j} 2 \pi k / N} z^{-1}}
$$




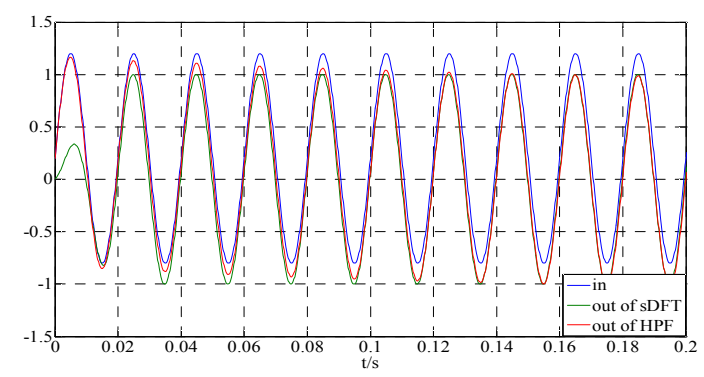

Fig. 3. Simulation results of $\operatorname{sDFT}(N=400)$ and $\operatorname{HPF}\left(f_{\mathrm{c}}=1 \mathrm{~Hz}\right)$.

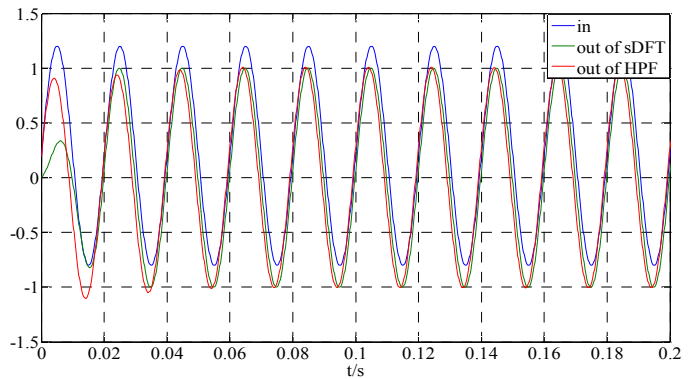

Fig. 4. Simulation results of $\operatorname{sDFT}(N=400)$ and $\operatorname{HPF}\left(f_{\mathrm{c}}=10\right.$ $\mathrm{Hz})$.

\section{B. Comparison between DFT and HPF}

Sliding DFT and DFT (which can be taken as a main value sequence of DFS) exhibit similar characteristics with the sole difference being implementation speed. The ratio of calculation amount between DFT and sDFT is $\left(\log _{2}{ }^{N}\right) / 2$, provided the same data number $N$ [12].

HPF, in which cut-off frequency, order, and type crucially impact the filter dynamic response process and the estimation precision, can restrain dc bias along with a simple implementation [15]. For example, a low cut-off frequency is beneficial to improve the estimation accuracy for a 2-order Butterworth HPF. However, the dynamic response slows down because of the large time delay. Thus, although a high cut-off frequency can speed up the dynamic estimation process, such frequency will cause waveform distortion and affect the estimation accuracy.

We assume an input signal $x_{0}=\sin \left[\left(2 * \pi * f_{0}\right) * t\right]+0.2$, where $f_{0}=50 \mathrm{~Hz}$, and 0.2 is the dc bias. The fundamental component of the input signal extracted by SDFT and HPF are compared using MATLAB.

As regards sDFT, the sampling frequency $f_{\mathrm{s}}=5 \mathrm{kHz}$, and the data number $N=f_{\mathrm{s}} / f_{0}=400$. The cut-off frequency of HPF is set at different values $\left(f_{\mathrm{c}}=1 \mathrm{~Hz}, f_{\mathrm{c}}=10 \mathrm{~Hz}\right.$, and $\left.f_{\mathrm{c}}=20 \mathrm{~Hz}\right)$. The simulation waveforms are shown in Figs. 3, 4, and 5.

Figs. 3 to 5 illustrate that SDFT and HPF can restrain the $\mathrm{dc}$ bias within one fundamental period. However, when $f_{\mathrm{c}}=1$ $\mathrm{Hz}, \mathrm{HPF}$ tracks performance precisely at a steady state with the dynamic adjustment time at nearly six to eight fundamental periods, as shown in Fig. 3. Although increasing $f_{\mathrm{c}}$ can accelerate the dynamic process, this condition can result in low precision, as shown in Figs. 4 and 5.

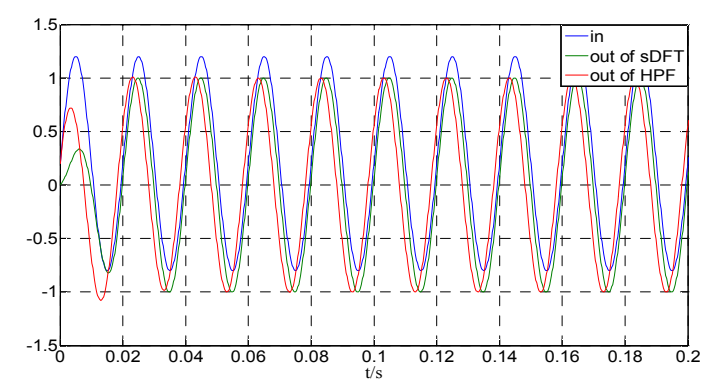

Fig. 5. Simulation results of $\operatorname{sDFT}(N=400)$ and $\operatorname{HPF}\left(f_{\mathrm{c}}=20\right.$ $\mathrm{Hz})$.

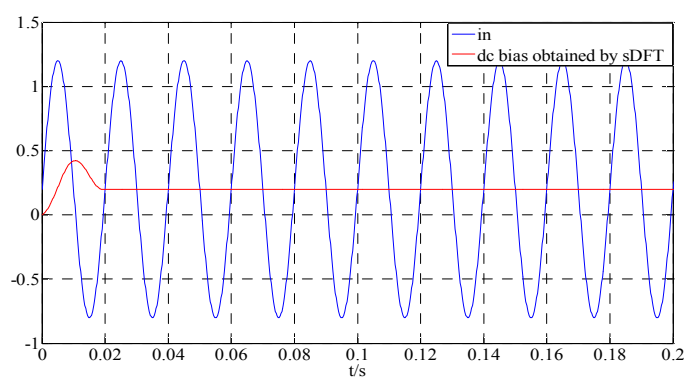

Fig. 6. DC bias obtained by $\operatorname{sDFT}(N=400)$.

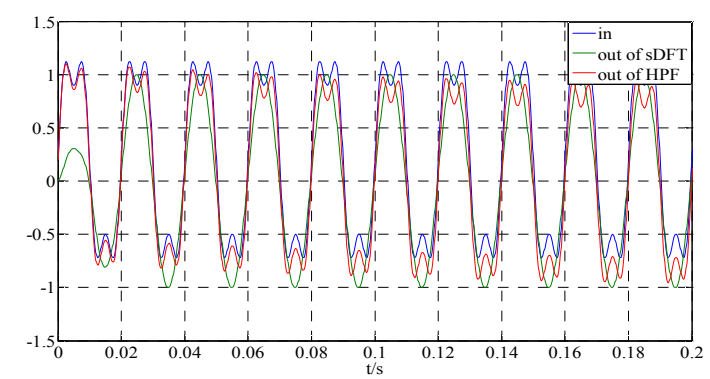

Fig. 7. Simulation results of $\operatorname{sDFT}(N=400, k=1)$ and $\operatorname{HPF}\left(f_{\mathrm{c}}=\right.$ $1 \mathrm{~Hz}$ ) with a harmonic input.

In addition, sDFT can extract harmonic or dc bias components any time by setting different $k$ values in Equation (11). The dc bias in input signal $x_{0}$ obtained by SDFT is shown in Fig. 6. When $x_{0}=$ $\sin \left[\left(2 * \pi^{*} f_{0}\right) * t\right]+0.3 * \sin \left[\left(6 * \pi * f_{0}\right) * t\right]+0.2, \quad$ the estimation results of $\operatorname{sDFT}(N=400, k=1)$ and $\operatorname{HPF}\left(f_{\mathrm{c}}=1 \mathrm{~Hz}\right)$ are shown in Fig. 7.

\section{APPROACH TO ESTIMATE THE ROTOR INITIAL POSITION BASED ON SDFT}

The flux obtained from the integral voltage model can be regarded as finite sequences. We define sequences $\psi_{0}(n)$, $\psi_{1}(n)$ as $x_{0}(n)$ and $x_{1}(n)$ in Fig. 1, and their DFTs are

$$
\begin{aligned}
\Psi_{0}(k)= & \psi(0)+\psi(1) e^{-\mathrm{j} \frac{2 \pi k}{N}}+\psi(2) e^{-\mathrm{j} \frac{2 \pi k * 2}{N}}+\cdots \\
& +\psi(N-1) e^{-\mathrm{j} \frac{2 \pi k^{*}(N-1)}{N}}
\end{aligned}
$$




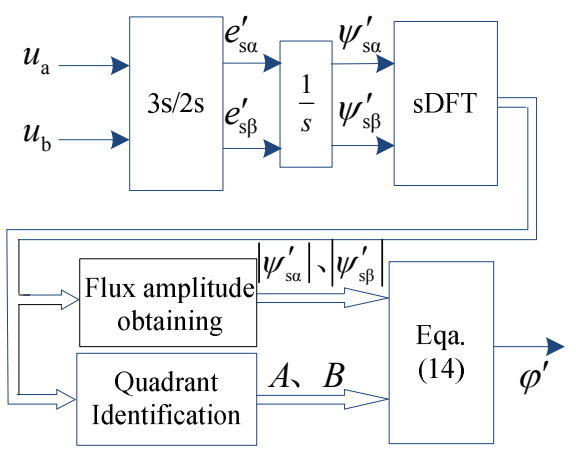

Fig. 8. Estimation principle.

TABLE I

PosSible QuAdRANT WITH THE DifFERENT Rotor INITIAL POSITIONS

\begin{tabular}{|c|c|c|}
\hline Flux sign & Quadrant & Identifiers \\
$\psi_{\mathrm{s} \alpha}^{\prime}>0, \psi_{\mathrm{s} \beta}^{\prime}>0$ & First quadrant & $A=0, B=1$ \\
$\psi_{\mathrm{s} \alpha}^{\prime}<0, \psi_{\mathrm{s} \beta}^{\prime}>0$ & Second quadrant & $A=1, B=-1$ \\
$\psi_{\mathrm{s} \alpha}^{\prime}<0, \psi_{\mathrm{s} \beta}^{\prime}<0$ & Third quadrant & $A=-1, B=1$ \\
$\psi_{\mathrm{s} \alpha}^{\prime}>0, \psi_{\mathrm{s} \beta}^{\prime}<0$ & Four quadrant & $A=0, B=-1$ \\
\hline
\end{tabular}

$$
\begin{aligned}
\Psi_{1}(k) & =\left[\Psi_{0}(k)-\psi(0)\right] e^{\mathrm{j} \frac{2 \pi k}{N}}+\psi(N) e^{-\mathrm{j} \frac{2 \pi k(N-1)}{N}} \\
& =\left[\Psi_{0}(k)-\psi(0)+\psi(N)\right] e^{\mathrm{j} \frac{2 \pi k}{N}}
\end{aligned}
$$

where $\Psi_{0}(k)$ and $\Psi_{1}(k)$ are complex components. When $k=1$, $\Psi_{0}(1)$ and $\Psi_{1}(1)$ represent the fundamental components, which can be decomposed into the real and imaginary components, through which the fundamental components $\psi_{\mathrm{s} \alpha}^{\prime}$, $\psi_{\mathrm{s} \beta}^{\prime}$ and their amplitudes $\left|\psi_{\mathrm{s} \alpha}^{\prime}\right|,\left|\psi_{\mathrm{s} \beta}^{\prime}\right|$, as well as their angle atan $2\left(\left|\psi^{\prime}{ }_{\mathrm{s} \alpha}\right|,\left|\psi_{\mathrm{s} \beta}^{\prime}\right|\right)$, can be obtained.

Induced voltage and flux possess sine characteristics because of sine excitation. Given that the flux signs pre- and post-zero are opposite, a quadrant discrimination model is designed to avoid the zero drift, which will affect the direct arctangent calculation. First, we sample the fundamental flux $\psi_{\mathrm{s} \alpha}^{\prime}, \psi_{\mathrm{s} \beta}^{\prime}$ in the first half of the cycle. Then, the quadrant can be determined according to the flux sign (plus or minus), as well as the identifiers, $A$ and $B$. The possible quadrant with different rotor initial positions is shown in Table I.

The rotor initial position $\varphi^{\prime}$ can be calculated by using Equation (14).

$$
\varphi^{\prime}=A \cdot \pi+B \cdot \operatorname{atan} 2\left(\left|\psi_{\mathrm{s} \alpha}^{\prime}\right|,\left|\psi_{\mathrm{s} \beta}^{\prime}\right|\right)
$$

The detailed implementation for the rotor initial position estimation proposed in this paper is shown in Fig. 8, and the flow chart of the implementation is shown in Fig. 9.

\section{SimULATION AND EXPERIMENTAL RESULTS}

\section{A. Simulation Results}

A simulation was established in MATLAB. In this simulation, the rotor initial position was set at $60^{\circ}$ and the dc

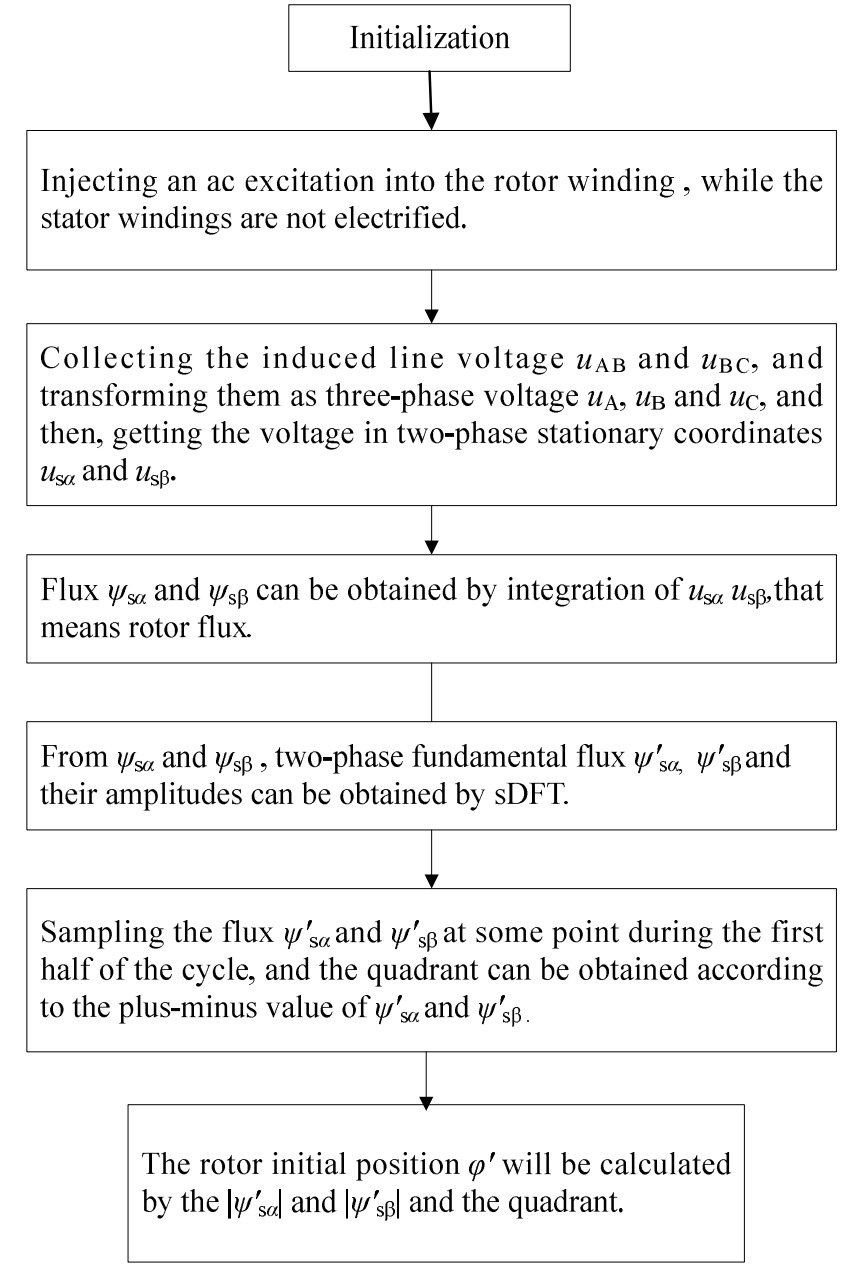

Fig. 9. Implementation chart of the program.

bias errors of the induced stator voltage $u_{\mathrm{a}}$ and $u_{\mathrm{b}}$ were 0.3 and $0.5 \mathrm{~V}$, respectively. When ac excitation was injected into the rotor winding, the induced voltage in stator windings are shown in Fig. 10(a), whereas the flux in the $\alpha \beta$ coordinates obtained through the integral voltage model are presented in Fig. 10(b). The fundamental components of the induced flux calculated by sDFT are shown in Fig. 10(c), whereas their amplitudes are shown in Fig. 10(d). The estimated rotor initial position is presented in Fig. 10(e).

This kind of estimation method can be applied once dc excitation is injected into the rotor winding. Another simulation was established in MATLAB with the same configurations, and the fundamental components of the induced flux calculated by sDFT are shown in Fig. 11(a). The estimated rotor initial position is shown in Fig. 11(b).

Figs. 10 and 11 suggest that, the rotor initial position estimation method based on sDFT is suitable for either ac or dc injection. Furthermore, a dc current can result in the rapid attenuation of the induced stator voltage and flux, similar to the comparison between Figs. 10(c) and Fig. 11(a), which resulted in the estimation deviation shown in Fig. 11(b). 


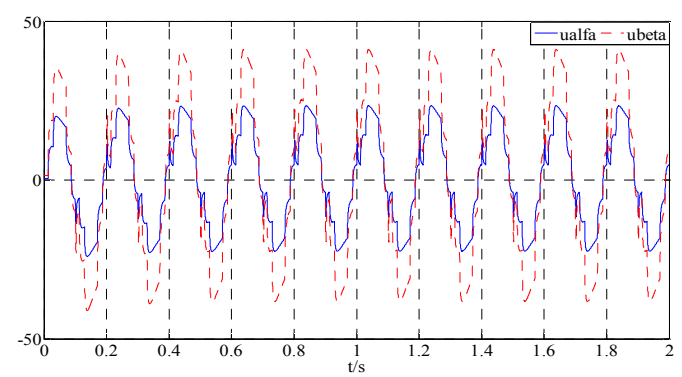

(a) Induced stator voltage.

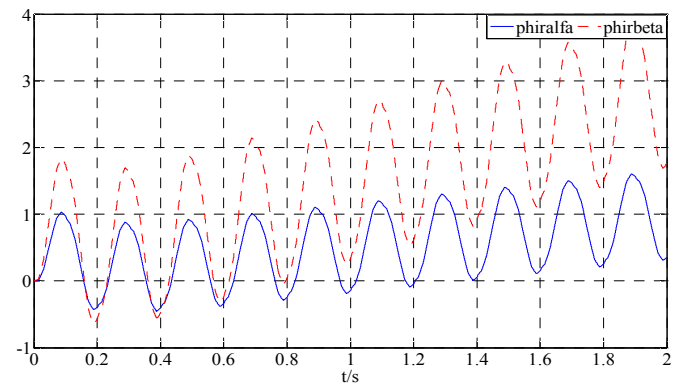

(b) Stator flux by an integral voltage model.

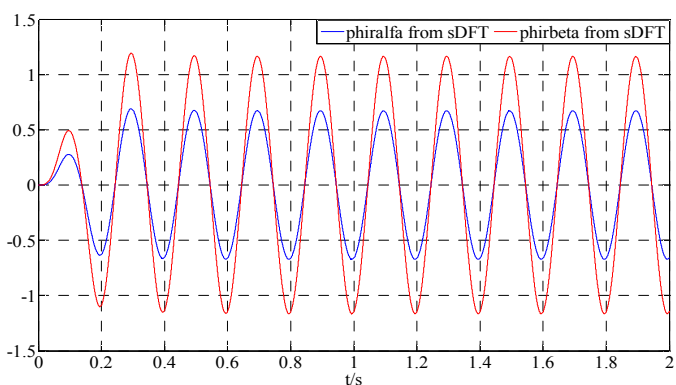

(c) Fundamental flux obtained from sDFT.

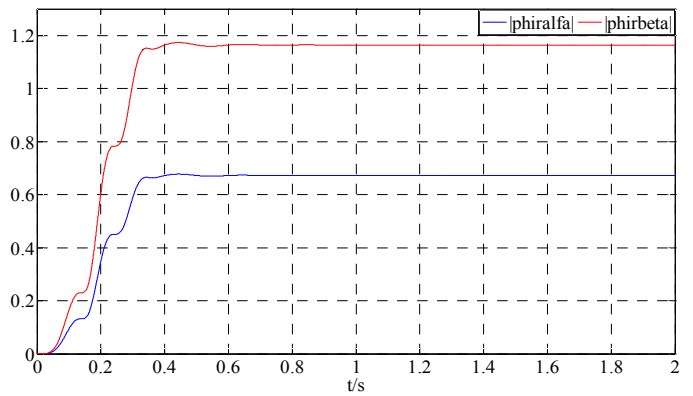

(d) Corresponding amplitudes.

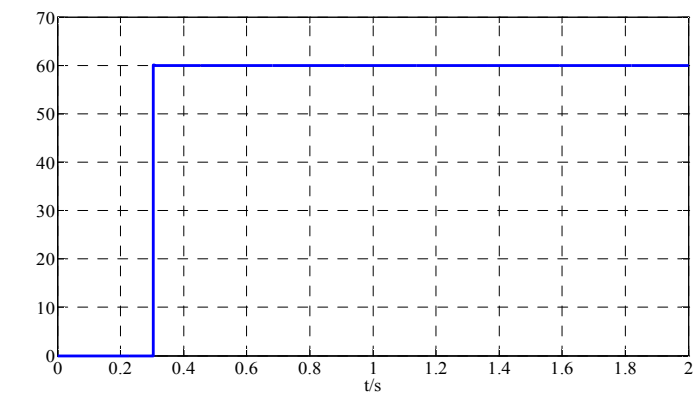

(e) Rotor initial position.

Fig. 10. Simulation results based on sDFT method with ac current injection.

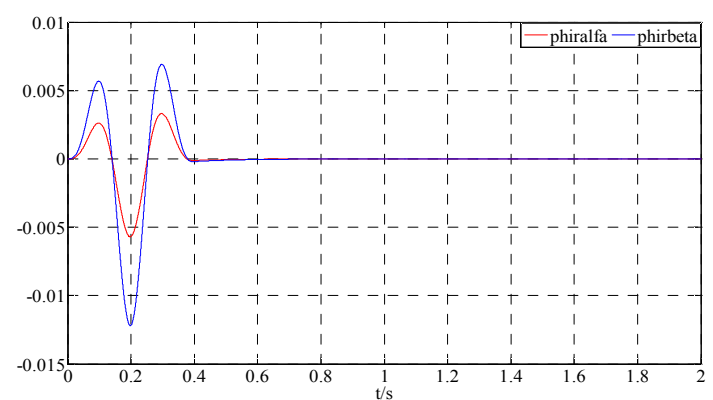

(a) Fundamental flux obtained from sDFT

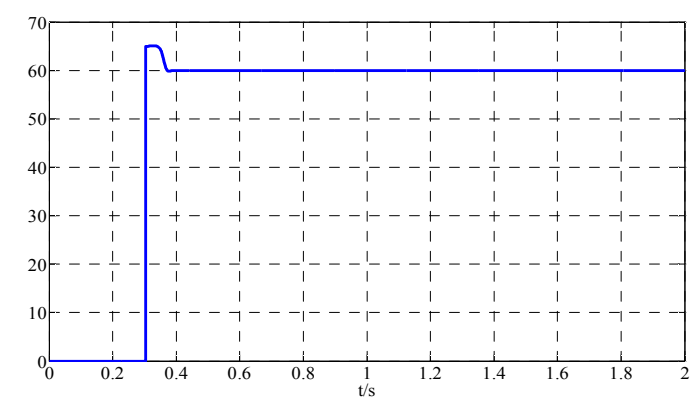

(b) Rotor initial position

Fig. 11. Simulation results based on sDFT method with dc current injection.

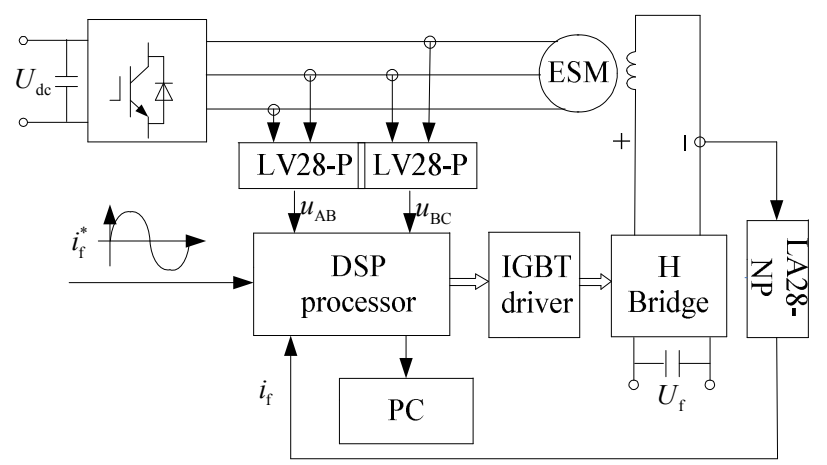

Fig. 12. Circuit block diagram of the rotor initial position estimation with voltage sensors.

\section{B. Experimental Results}

An experimental platform for a $380 \mathrm{~V}, 50 \mathrm{~kW}$ EESM was established to verify the effectiveness of the proposed estimation method. The experimental structure is shown in Fig. 12, whereas the detailed parameters of EESM are shown in Table II.

Common voltage sensors can induce high-frequency interference and quantization error during the sampling process, which affect estimation accuracy. In this paper, we used an oscilloscope (DPO3014) to monitor the induced voltage in stator windings and calculated real-time data using a digital signal proceeding (DSP) processor. The experimental structure without voltage sensors is shown in Fig. 13. 
TABLE II

Detailed PARAmeters of EESM

\begin{tabular}{|c|c|}
\hline Rated Power & $50 \mathrm{~kW}$ \\
Rated voltage & $380 \mathrm{~V}$ \\
Rated frequency & $50 \mathrm{~Hz}$ \\
Rated speed & $1500 \mathrm{rpm}$ \\
Rated power factor & 0.9 \\
Rated stator current & $84.41 \mathrm{~A}$ \\
Rated exciting current & $6.721 \mathrm{~A}$ \\
Stator resistance & $0.059 \Omega$ \\
Direct axis reaction reactance & $2.38 \mathrm{pu}$. \\
Quadrature axis reaction reactance & $1.22 \mathrm{pu}$. \\
Exciting resistance & $9.276 \Omega$ \\
Stator leakge reactance & $0.121 \mathrm{pu}$. \\
\hline
\end{tabular}

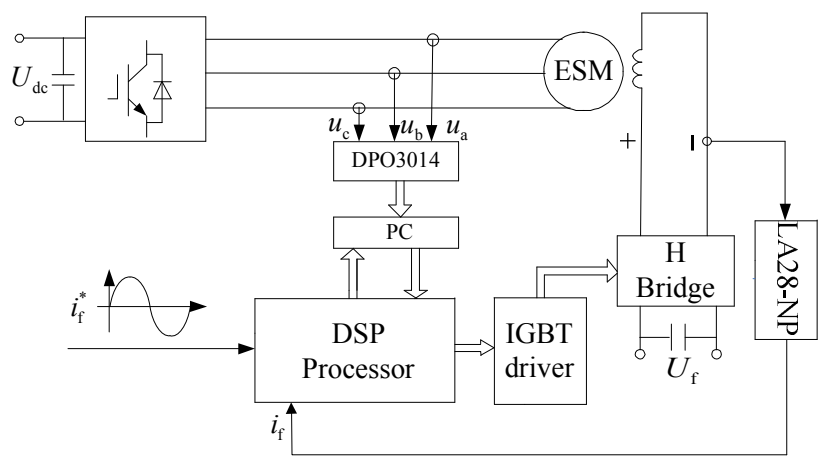

Fig. 13. Circuit block diagram of the rotor initial position estimation without voltage sensors.

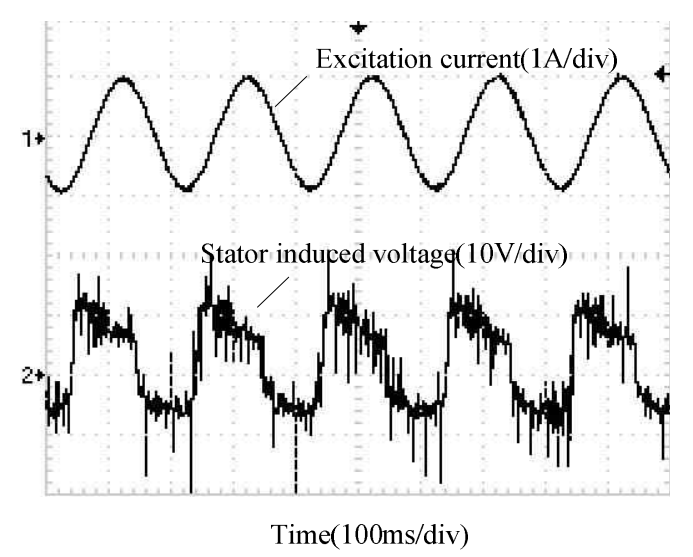

Fig. 14. Waveforms of the excitation current and stator induced voltage.

During the experiment, the peak-peak value of the ac excitation current is $1 \mathrm{~A}$, with a $5 \mathrm{~Hz}$ frequency and $128 \mathrm{sDFT}$ points. The excitation current and the induced voltage in a-phase are shown in Fig. 14.

Fig. 15 shows the fundamental flux $\psi_{s \alpha}^{\prime}$ and $\psi_{s \beta}^{\prime}$ obtained through sDFT when the real rotor position is $60^{\circ}$. The estimated rotor initial position is shown in Fig. $16(\mathrm{CH} 1)$, and the $(\mathrm{CH} 2)$ illustrates the zero drift during flux crossing.

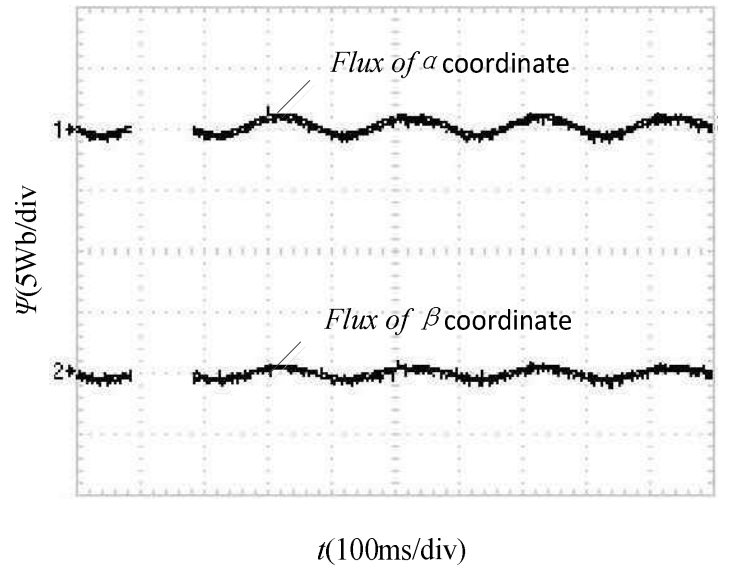

Fig. 15. Waveforms of the fundamental stator flux by sDFT.

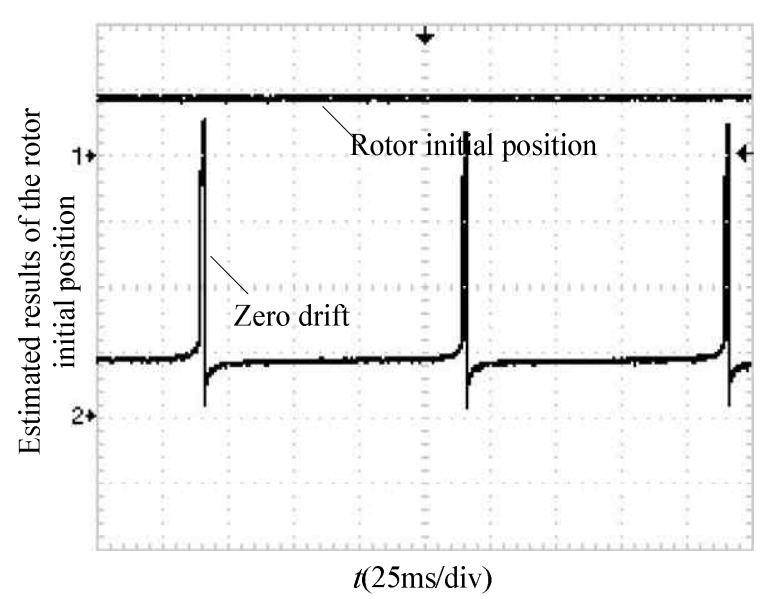

Fig. 16. Initial rotor position detected by sDFT.

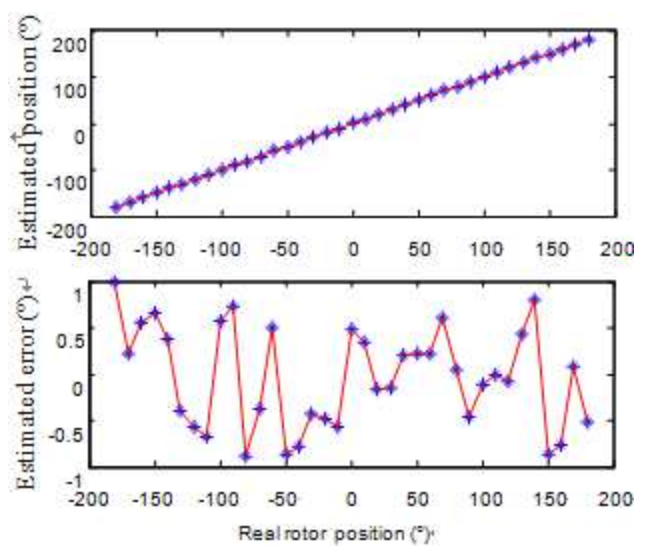

Fig. 17. Results of the initial rotor position estimation.

Fig. 17 shows the experimental results when the real rotor position is horizontal coordinate and the estimated position error is vertical. This presents an estimation error within $\pm 1^{\circ}$ as well.

The real process of the estimation method applied for the starting process of EESM is shown in Fig. 18. The estimation section for the rotor initial position occurred when $t=1 \mathrm{~s}$ to 


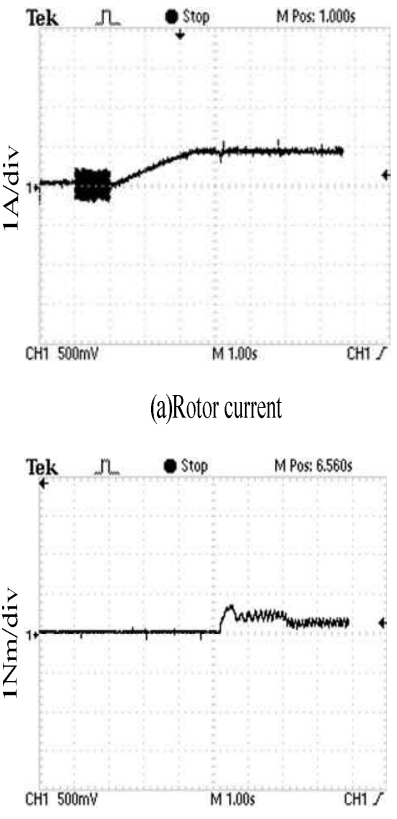

(c)Electromagnetic torque

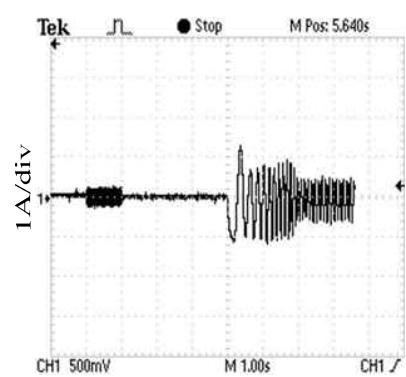

(b)Phase A current

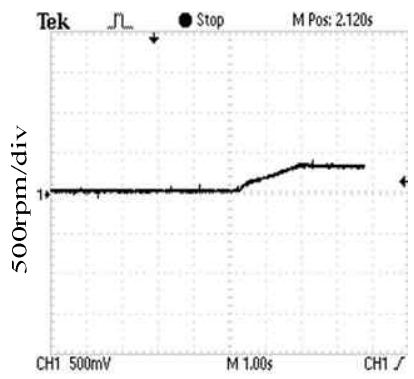

(d)Motor speed
Fig. 18. Starting process of the EESM.

$2 \mathrm{~s}$, and the ac excitation injected into the rotor winding was established during $t=2 \mathrm{~s}-5 \mathrm{~s}$. When $t=5 \mathrm{~s}$ to $7 \mathrm{~s}$ was the accelerating process and the machine was maintained at 300 rpm after $t=7 \mathrm{~s}$. These experimental results verify the effectiveness of the improved estimation method, which can be applied to the actual drive system.

\section{CONCLUSIONS}

This paper presented a novel estimation method for the rotor initial position of EESMs based on sDFT. First, an induced voltage was generated in the stator windings by injecting ac excitation into the rotor winding. Second, the induced stator flux resulted from the integral voltage model, which caused such problems as dc bias and initial value of integration. Third, the fundamental component of the induced flux was obtained through sDFT, with which the rotor initial position was estimated.

This proposed method possessed a simplified structure, easy implementation, strong anti-interference, and minimal hardware consumption. Comparisons between sDFT and HPF, with ac and dc excitations, were conducted. Experimental results verified the effectiveness of the proposed method.

\section{ACKNOWLEDGMENT}

The authors would like to thank the China National Natural Science Foundation (51377160) and the Research and Innovation Program of Postgraduates in the Jiangsu Province (CXZZ12_0930).

\section{REFERENCES}

[1] X. L. Ma, High Performance Frequency Speed Regulation and the Typical Control System, China Machine Press, chap. 8, 2010.

[2] J. A. Pontt, J. R. Rodriguez, A. Liendo, and P. Newman, "Network friendly low switching frequency multipulse high power three level PWM rectifier," IEEE Trans. Ind. Electron., Vol. 56, No. 4, pp. 1254-1262, Apr.2009.

[3] Y. Z. Zhou, Y. W. Hu, and W. X. Huang, "Research on a direct torque control for electrically excited synchronous motor drive with low ripple in flux and torque," in Proc. the CSEE, Vol. 26, No. 7, pp. 152-157, 2006.

[4] Q. Q. Yuan, X. J. Wu, X. Fu and P. Dai, "Control of electrically excited synchronous motors with a low switching frequency," Journal of power electronics, Vol. 12, No. 4, pp.615-622, Jul. 2012.

[5] T. Chun and Y. W. Hu, "A novel torque control in electromagnetic synchronous motor drive," Journal of Nanjing University of Aeronautics and Astronautics, Vol. 33, No. 2, pp. 108-112, Apr. 2001.

[6] H. S. Jung, S. H. Hwang, and J. M. Kim., "Diminution of current measurement error for vector-controlled AC motor drives," IEEE Trans. Ind. Appl., Vol. 42, No. 5, pp. 1249-1256, Sep./Oct. 2006.

[7] Y. Z. Zhou and Y. W. Hu, "A novel direct torque control for electrically excited synchronous motor drives with high power factor and low ripples in flux and torque," in IEEE Power Electronics Specialists Conference, pp. 4752-4756, 2008.

[8] K. R. Cho and J. K. Seok, "Pure-integration-based flux acquisition with drift and residual error compensation at a low stator frequency," IEEE Trans. Ind. Appl., Vol. 45, No. 4, pp. 1276-1285, Jul./Aug. 2009.

[9] N. Bianchi, S. Bolognani, J. H. Jang, and S. K. Sul, "Advantages of inset PM machines for zero-speed sensorless position detection," IEEE Trans. Ind. Appl., Vol. 44, No. 4, pp.1190-1198, Jul./Aug. 2008.

[10] J. H. Jang, J. I. Ha, M. Ohto, and S. K. Sul, "Analysis of permanent-magnet machine for sensorless control based on high-frequency signal injection," IEEE Trans. Ind. Appl., Vol. 40, No. 6, pp.1595-1604, Nov./Dec. 2004.

[11] X. M. Deng, L. Wang, J. M. Zhang, and Z. X. Ma, "Rotor position detection of synchronous motor based on high-frequency signal injection into the rotor," in 2011 Third International Conference on Measuring Technology and Mechatronics Automation, pp.195-198, 2011.

[12] E.Jacobsen and R. Lyons, "The sliding DFT," IEEE Signal Process. Mag, Vol. 20, No. 2, pp. 74-80, Mar. 2003.

[13] S. Kouro, B. L. Rocca, P. Cortes, and S. Alepuz, "Predictive control based selective harmonic elimination with low switching frequency for multilevel converters," in 2009 Energy Conversion Congress and Exposition, pp. 3130-3136, 2009.

[14] E. Jacobsen and R. Lyons, "An update to the sliding DFT", IEEE Signal Process. Mag, Vol. 21, No. 1, pp.110-111, Jan. 2004.

[15] Q. Wang, W. Z. Yao, and Z. A. Wang, "A study about influence of high and low pass filter on detecting effect of harmonics detection circuits," Transactions of China Electrotechnical Society, Vol. 14, No. 5, pp. 22-26, Oct. 1999. 


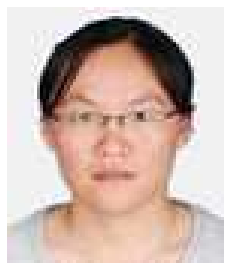

Qing-qing Yuan was born in NanTong, JiangSu, China in 1987. She received her B.S and M.S. from the China University of Mining and Technology, China, in 2009 and 2011, respectively. She began her Ph.D. program in 2011 with the Department of Information and Electrical Engineering, China University of Mining and Technology. Her current research interests include the modeling and control of high power drives with a low switching frequency.

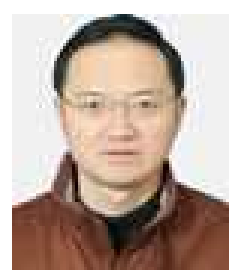

Xiao-jie Wu was born in HengYang, HuNan, China, in 1966. He received his B.S. in Industrial Automation from the China University of Mining and Technology, China, in 1988, and his M.S. and Ph.D. in Electrical Engineering from the China University of Mining and Technology, China, in 1991 and 2000, respectively. From 2002 to 2004, he conducted postdoctoral research at Tsinghua University, Beijing, China. He has been with the Department of Information and Electrical Engineering, China University of Mining and Technology, China, since 1991, where he is currently a Professor. His current research interests include the stability of ac machines, advanced control of electrical machines, and power electronics.

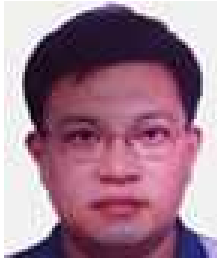

Peng Dai was born in HuaiBei, AnHui, China, in 1973. He received his B.S. in Electrical Engineering from the AnHui University of Science and Technology, Huainan, China, in 1994, and his M.S. and Ph.D. in Electrical Engineering from the China University of Mining and Technology, China, in 1998 and 2006, respectively. He has been with the Department of Information and Electrical Engineering, China University of Mining and Technology, China, since 1998, where he is currently a Professor. His current research interests include the stability and control of synchronous machines. 